\title{
A Comparison of Visceral and Somatic Pain Processing in the Human Brainstem Using Functional Magnetic Resonance Imaging
}

\author{
Paul Dunckley, ${ }^{1,2}$ Richard G. Wise, ${ }^{1,2}$ Merle Fairhurst, ${ }^{1,2}$ Peter Hobden, ${ }^{2}$ Qasim Aziz, ${ }^{3}$ Lin Chang, ${ }^{4}$ and Irene Tracey ${ }^{1,2}$ \\ ${ }^{1}$ Department of Human Anatomy and Genetics and ${ }^{2}$ Centre for Functional Magnetic Resonance Imaging of the Brain, University of Oxford, Oxford OX1 \\ 3QX, United Kingdom, ${ }^{3}$ Department of GI Science, University of Manchester, Hope Hospital, Salford M6 8HD, United Kingdom, and ${ }^{4}$ Center for \\ Neurovisceral Sciences and Women's Health, Division of Digestive Diseases, David Geffen School of Medicine at University of California Los Angeles, Los \\ Angeles, California 90073
}

\begin{abstract}
Evidence from both human and animal studies has demonstrated a key role for brainstem centers in the control of ascending nociceptive input. Nuclei such as the rostral ventromedial medulla and periaqueductal gray (PAG) are able to both inhibit and facilitate the nociceptive response. It has been proposed that altered descending modulation may underlie many of the chronic pain syndromes (both somatic and visceral). We used functional magnetic resonance imaging to image the neural correlates of visceral and somatic pain within the brainstem. Ten healthy subjects were scanned twice at 3 tesla, during which they received matched, moderately painful, electrical stimuli to either the midline lower abdomen or rectum. Significant activation was observed in regions consistent with the PAG, nucleus cuneiformis (NCF), ventral tegmental area/substantia nigra, parabrachial nuclei/nucleus ceruleus, and red nucleus bilaterally to both stimuli. Marked spatial similarities in activation were observed for visceral and somatic pain, although significantly greater activation of the NCF (left NCF, $p=0.02$; right NCF, $p=0.01$; Student's paired $t$ test, two-tailed) was observed in the visceral pain group compared with the somatic group. Right PAG activity correlated with anxiety during visceral stimulation $(r=0.74 ; p<0.05$, Pearson's $r$, two-tailed $)$ but not somatic stimulation. We propose that the differences in NCF and right PAG activation observed may represent a greater nocifensive response and greater emotive salience of visceral over somatic pain.
\end{abstract}

Key words: visceral; somatic; fMRI; rectum; pain; brainstem

\section{Introduction}

Since 1906, we have known that spinal cord excitability is directly influenced by descending, tonically active signals that are mostly inhibitory in function. Work by Sherrington (1906) showed that nociceptive reflexes were enhanced after the spinal cord was transected. This supraspinal descending modulation was demonstrated graphically by World War I soldiers with severe injuries who reported disproportionately low pain sensation (Beecher, 1946), presumably because of mechanisms of distraction or stress-induced analgesia. Distraction from pain decreases both the intensity and affective components of the pain experience (Miron et al., 1989; Bantick et al., 2002). Therefore, cortical and subcortical circuitry exists to allow pain modulation in a top-

\footnotetext{
Received March 21, 2005; revised June 21, 2005; accepted June 21, 2005.

P.D. and L.C. are funded through Grant AR41622 from the National Institute of Arthritis and Musculoskeletal and Skin Diseases-National Institutes of Health (Bethesda, MD). R.G.W. is funded through Advanced Training Fellowship 067037 from The Wellcome Trust. Q.A. is funded by the Medical Research Council and Cancer Research Campaign (London, UK). I.T. is funded by the Higher Education Funding Council for England and Medical Research Council of Great Britain and Northern Ireland (FMRIB Centre). We acknowledge the Royal Society for an equipment grant (RSRG 24647). We thank Andreas Schoenfeld of Standard Instruments (Karlsruhe, Germany) for technical support. We also thank Prof. Derek Jewell for his guidance and support.

Correspondence should be addressed to Dr. Irene Tracey, Department of Human Anatomy and Genetics, South Parks Road, 0xford 0X130X, UK. E-mail: irene.tracey@human-anatomy.oxford.ac.uk.

DOI:10.1523/JNEUROSCI.1100-05.2005

Copyright $\odot 2005$ Society for Neuroscience $\quad$ 0270-6474/05/257333-09\$15.00/0
}

down manner. Over the last 35 years, an accumulation of evidence has demonstrated key roles for brainstem centers in this control of nociception. In a pivotal study, Reynolds (1969) highlighted the role of the periaqueductal gray (PAG) in the nocifensive response: electrical stimulation of the PAG in rats allowed abdominal surgery without the use of general anesthesia. This inhibition of the normal pain response through stimulation of discrete brainstem centers such as the PAG [stimulus-produced analgesia (SPA)] was subsequently described in humans (Boivie and Meyerson, 1982; Baskin et al., 1986).

In animals, SPA has also been demonstrated in response to stimulation of the rostral ventromedial medulla (RVM) (Basbaum et al., 1976; Fields et al., 1977), which is situated centrally around the pontomedullary junction. Exposure of this region to both higher intensities of electrical stimulation and higher concentration of stimulatory neurotransmitters can induce analgesia (Zhuo and Gebhart, 1990; Gebhart, 2004). The RVM has a dual role in pain control: it is able to both inhibit and facilitate the nociceptive input. The latter is achieved through low-intensity electrical stimulation or low concentrations of stimulatory neurotransmitters. Facilitation of pain via the RVM has been implicated in the development of central sensitization and secondary hyperalgesia (Urban and Gebhart, 1997, 1999). It has been proposed therefore that disordered descending influences from the 
brainstem on nociceptive afferent information may underlie abnormal pain perception in both functional pain disorders, such as irritable bowel syndrome and fibromyalgia, and neuropathic pain (Porreca et al., 2002).

To establish whether aberrant brainstem pain processing underlies human chronic somatic or visceral pain syndromes, noninvasive methods of investigating brainstem function need to be developed. Technical difficulties in functionally imaging the brainstem, such as poor spatial resolution, local field inhomogeneity-induced signal losses, and image distortion have precluded any pain study in humans. For a review of these issues and of which studies to date have identified brainstem activation using neuroimaging methods, see Tracy and Iannetti (2005). Only a few studies to date have successfully identified regional brainstem activity in individual subjects during pain, fine touch, swallow, motor activities and taste (Komisaruk et al., 2002; Tracey et al., 2002; Topolovec et al., 2004). The aims of this study were to compare the regional brainstem activation between visceral and somatic pain. We therefore performed high-resolution functional magnetic resonance imaging (fMRI) in healthy subjects during both rectal and lower abdominal electrical stimulation matched for pain intensity and unpleasantness.

\section{Materials and Methods}

Subjects. Ten healthy, right-handed subjects (five female) participated in the study. The median age was 30 years of age (range, $21-33$ years). None reported any abnormal gastrointestinal or somatic symptoms on a personal history and bowel symptom questionnaire (Talley et al., 1989). Clinical depression was excluded with the use of the Beck's Depression Inventory (Beck et al., 1961). None of the subjects took any medications likely to interfere with the blood oxygen level-dependent response. All subjects gave informed consent, and the Oxfordshire Clinical Research Ethics Committee approved the study.

Stimuli. Electrical stimuli were used to induce both somatic and visceral pain. Purpose-built electrical stimulating catheters were designed for safe use within the high field strength magnet (Standard Instruments, Karlsruhe, Germany). These contained no ferromagnetic material and had two bipolar ring electrodes $2 \mathrm{~cm}$ apart at the distal tip. The catheters were connected via a magnet-compatible extension cable to a Digitimer (Welwyn Garden City, UK) DS7A constant current stimulator. The DS7A automatically varies the voltage depending on the impedance in the circuit to provide a constant, predefined current. A Digitimer DG2 trigger generator was used to provide $6 \mathrm{~s}$ trains of electrical pulses delivered at a frequency of $5 \mathrm{~Hz}$.

To induce visceral pain, the catheters were inserted into the rectum, 10 $\mathrm{cm}$ from the anal canal. For somatic pain, the catheters were attached to the lower abdomen, in the midline at the dermatome level of T12.

Study design. Before the functional scan, increasing $6 \mathrm{~s}$ stimuli were delivered to the subject in a stepwise manner to define the current required to induce a reliable pain intensity rating of 5 out of 10 . The subjects were instructed that a rating of 0 meant no pain and a rating of 10 represented excruciating pain. This current was used for the remainder of the experiment. During the functional scan, 20 6-s stimuli were delivered to the subject with an average $60 \mathrm{~s}$ interstimulus interval. The subjects rated each stimulus via a numerical rating scale projected onto a screen that was visualized via prism glasses. An arrow on the scale could be moved via a button box. Unpleasantness was also rated ( 0 , not unpleasant; 10 , unbearably unpleasant) along with urge sensation ( 0 , no urge; 10 , intense urge). All scales were presented to the subjects during the interstimulus interval. The order of the visceral and somatic scans was randomized. Subjects were then asked to retrospectively rate their average anxiety levels during the stimuli (0, no anxiety; 10, highly anxious).

Data acquisition. For the functional data, subjects were scanned in a 3 tesla human MRI system (Oxford Magnet Technology, Oxford, UK) with the use of a Magnex SGRAD MK III head coil (Magnex Scientific, Oxford, UK). To minimize the image distortion induced through B0 magnetic field inhomogeneities in the vicinity of the brainstem, coronal-

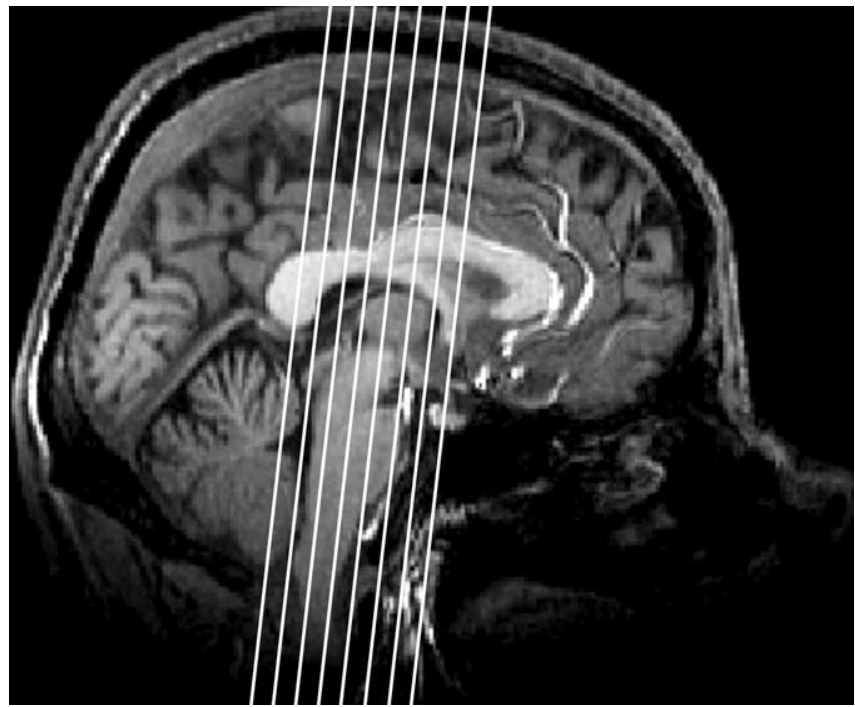

Figure 1. Graphical representation of the slice orientation during the functional scan. For clarity, the underlying image is a T1-weighted structural scan, and the number of slices was reduced from 24 to 8 . A coronal-oblique orientation was used with slices aligned with the ventral wall of the fourth ventricle.

oblique slices were acquired in the plane of the anterior wall of the fourth ventricle, graphically shown in Figure 1. Twenty-four $2 \mathrm{~mm}$ slices with an in-plane resolution of $3 \times 3 \mathrm{~mm}$ were obtained with the following parameters: repetition time (TR), $3 \mathrm{~s}$; echo time (TE), $30 \mathrm{~ms}$; flip angle, $90^{\circ}$; field of view, $192 \times 192 \mathrm{~mm}$; matrix, $64 \times 64$. These parameters were determined after a series of pilot functional scans. They resulted in the most reliable brainstem activation across subjects at a resolution that was high enough to be able to identify individual brainstem nuclei. The coverage was limited to the brainstem and a segment of cortex extending from the superior colliculus posteriorly to the hypothalamus anteriorly but incorporated the full width of the brain. To minimize B0 field inhomogeneity-induced effects, particular attention was given to optimal shimming before the commencement of the functional imaging run. A whole-brain structural proton-density turbo spin echo (PDTSE) scan was acquired in an equivalent coronal-oblique plane on a Siemens (Munich, Germany) Magnetom 1.5 T scanner. The parameters used were as follows: turbo factor, 9; effective TE, $12 \mathrm{~ms}$; TR, $6 \mathrm{~s}$; slice thickness, $2 \mathrm{~mm}$; in-plane resolution, $0.75 \times 0.75 \mathrm{~mm}$; three averages. To quantitatively evaluate the intensity of activation within individual brainstem nuclei, region of interest (ROI) masks were produced. To facilitate mask production, an additional structural, axially acquired PDTSE brainstem scan was obtained with maximal brainstem contrast (Fig. 2). Parameters for this scan were as follows: turbo factor, 3 ; effective TE, $12 \mathrm{~ms}$; TR, $5 \mathrm{~s}$; slice thickness, $3 \mathrm{~mm}$; in-plane resolution, $0.8 \times 0.8 \mathrm{~mm}$; six averages.

Image analysis. Image analysis, aimed at delineating significant brain activation via changes in the blood oxygen level-dependent signal (Ogawa et al., 1992), was performed on each subject's functional data set using the Expert Analysis Tool (www.fmrib.ox.ac.uk/fsl/) of the Centre for Functional Magnetic Resonance Imaging of the Brain (FMRIB) (Smith et al., 2001). Before the statistical analysis, brain segmentation was performed with the use of FMRIB brain-extraction tool (BET) (Smith, 2002). All scans were scrutinized after BET, procedure and additional manual segmentation was performed if required, thus optimizing the accuracy of any registrations. The data were motion corrected with the use of MCFLIRT (Jenkinson et al., 2002), spatial smoothing was performed with a Gaussian kernel of $4 \mathrm{~mm}$ full-width at half-maximum, intensity normalization was performed with a single scaling factor, and high-pass temporal filtering was performed with a Gaussian-weighted least-squares straight-line fit and a high-pass cutoff filter of $60 \mathrm{~s}$. The statistical analysis was performed with the improved linear model of FMRIB (Woolrich et al., 2001). A model of the relevant applied stimuli 

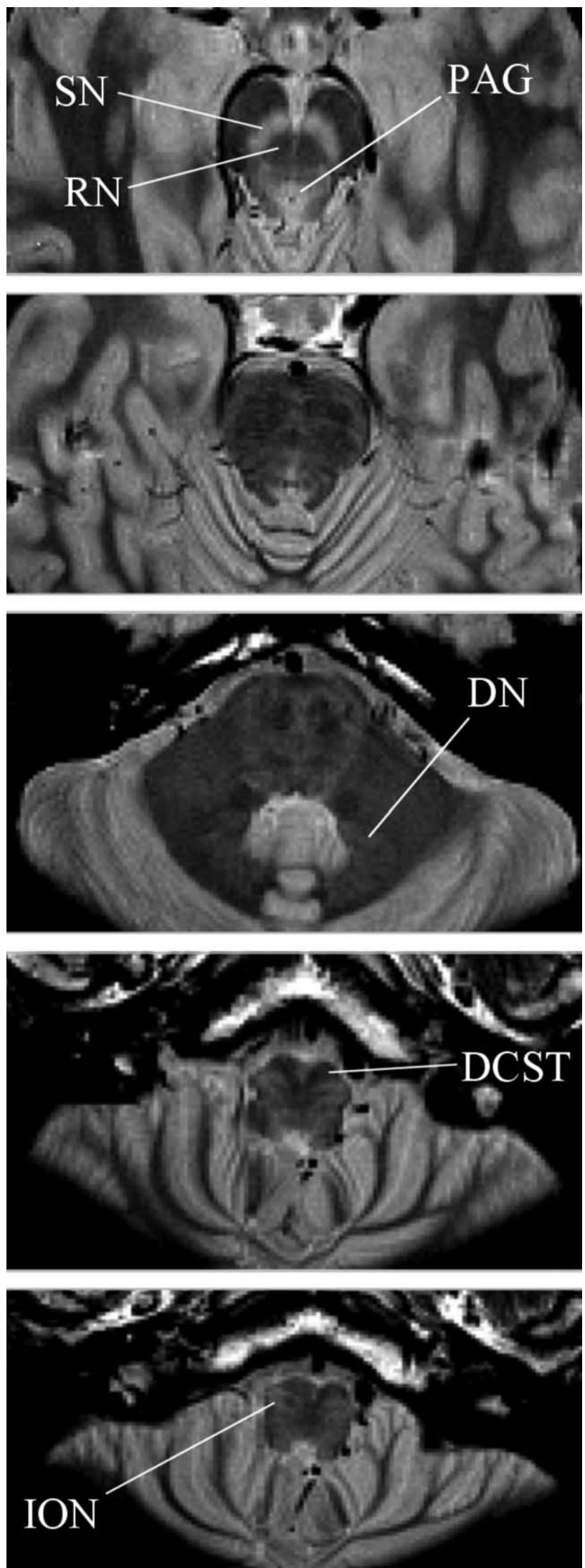

Figure 2. Axial slices through a PDTSE sequence structural scan of the brainstem. These were designed to provide maximal resolution and contrast within the brainstem to aid accurate region of interest mask formation. The substantia nigra (SN) and PAG are seen in lighter contrast. The red nucleus (RN), dentate nucleus (DN), decussation of the corticospinal tracts (DCST), and inferior olivary nucleus (ION) are labeled. was thus designed and convolved with the hemodynamic response function. This convolved model was then fitted to the four-dimensional data set to demonstrate areas of brain activation.

Each voxel was analyzed against the convolved model with a resultant parameter estimate (PE) image. The parameter estimate is proportional to the signal change arising from the stimulus. Cluster thresholding with significance estimation defined by Gaussian Random Field Theory was used to identify clusters of activated voxels $(z$-score $>2.3 ; p<0.01)$. The functional data set was coregistered onto the subject's high-resolution whole-brain PDTSE scan, which was then registered onto a standard brain [Montreal Neurological Institute (MNI) 152 brain] to facilitate group analysis.

A mixed-effects group analysis was performed for each of the two groups in standard brain space with Local Analysis of Mixed Effects (of the FMRIB). This incorporates variance within session and across time (fixed effects) and cross session variances (random effects). Cluster thresholding was performed with a $z$-threshold of 2.3 and corrected $p$ value of $<0.01$ (Worsley et al., 1992; Friston et al., 1994).

Left and right ROI masks were then defined for the PAG, RVM, nucleus cuneiformis (NCF), dorsolateral pons [the parabrachial nucleus $(\mathrm{PBN})$ and nucleus ceruleus $(\mathrm{NC})]$, and the ventral tegmental area (VTA). Because no stereotaxic atlas exists for the brainstem, the ROIs were defined by careful comparison with a detailed brainstem atlas (Duvernoy, 1995) using both T1-weighted structural scans and the axially acquired PDSE structural scan with optimal contrast within the brainstem (Fig. 2). The ROIs were then transformed into standard MNI space, and this transformed mask was applied to the individual's activation, also within standard MNI space. This method allows us therefore to take into account any subtle variations in brainstem anatomy between subjects. The mean PE was then calculated for each ROI for each subject's data set. Significant differences between the somatic and visceral mean group PE for each ROI were tested with a two-tailed paired Student's $t$ test (not corrected for multiple comparisons). Because the stimuli used were midline, we would expect any significant effects in the ROI analysis to be bilateral. This being the case, we contend that this reduces the chances of the bilateral effects we found being falsely reported as positive. Also these brainstem ROIs are part of a well recognized ascending and descending pain network and are not independent and often coactivate. Therefore, if the typical Bonferroni's correction were applied, it would be excessively stringent given its requirement for needing no a priori hypotheses and total independence of behavior between regions of interest. An independent analysis of the likelihood of finding bilateral significant differences of right and left NCF parameter estimates between the two stimulation paradigms by chance supports our reasoning and validity of results (data not shown). Correlation analysis of the mean parameter estimate between these regions was performed using Pearson's $r$ correlation analysis.

\section{Results}

\section{Psychophysical}

All subjects tolerated the study well. Electrical stimulation resulted in a reliably painful somatic and visceral sensation (Fig. 3). There were no significant differences in the intensity ratings or unpleasantness ratings between the two sensory modalities. Electrical stimulation of the rectum induced a mean urge of 1.3 (SEM, 0.58); however, six subjects reported no urge at all. A significantly greater strength of current was required to induce visceral pain of equal stimulus intensity to somatic pain (visceral, $23.1 \mathrm{~mA}$; SD, 21.3; somatic, $4.6 \mathrm{~mA}$; SD, 2.6; $p=0.02$; Student's $t$ test, twotailed). Anxiety ratings were obtained from eight subjects. The mean anxiety during visceral stimulation was significantly greater than somatic stimulation [visceral: mean rating, 4.56 (SD 1.24); somatic: mean rating, 2.69 (SD 1.87); $p=0.02$; Student's $t$ test, paired, two-tailed].

\section{Imaging}

A template, stereotaxic brain, such as the atlas by Talairach and Tournoux (1988), does not exist for the brainstem. Identification 


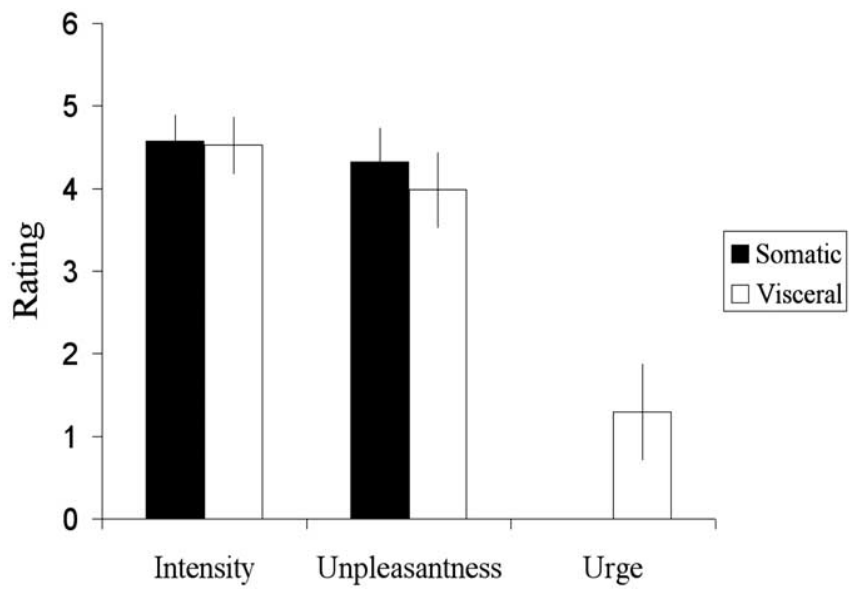

Figure 3. The group psychophysical data. The stimuli were well matched, with no significant differences in either intensity or unpleasantness rating between visceral or somatic stimulation ( $p>0.05$; Student's $t$ test, paired, two-tailed). Electrical stimulation of the rectum induced a mild urge sensation. Error bars represent SE.

of the specific regions activated therefore depended on careful identification of the areas of the brainstem with the use of a detailed accurate atlas (Duvernoy, 1995). Of the 10 subjects studied, activation within the brainstem (mesencephalon, pons, and/or medulla) occurred in seven subjects during both visceral and somatic pain (Table 1). In the remaining three subjects, brainstem activation was observed in just one of the two scans (two visceral and one somatic). The group activation maps for all 10 of the subjects are shown in Figure 4. A similar spatial pattern of activation can be seen for both sensory modalities. Bilaterally, the posterior insula cortex and thalamus (mediodorsal and ventrolateral thalamic nuclei) were activated in both groups, regions frequently activated in pain imaging studies.

In addition, there was concordance between both groups in the bilateral activation of the red nucleus, NCF, and PAG. An additional area of activation occurred bilaterally in the dorsolateral pons, anterolateral to the fourth ventricle. This region is consistent with locus ceruleus medially and parabrachial nuclei laterally and was common to both visceral and somatic pain. Both sensory modalities activated a region on the right side of the ventral mid pons. This area includes but extends beyond (mainly anteriorly) adrenergic center A5 in the visceral group but is situated more anteriorly in the somatic group. This peak of activation overlies the right pontine nuclei (PN). Left-sided PN activity was also seen in the visceral group. The $\mathrm{PN}$ act as relay points for the corticoponto-cerebellar pathway. These areas of activation are consistent with the projection from the primary and secondary sensory cortices (Brodal, 1968; Leergaard et al., 2004). A region in the inferior, anterior mesencephalon was activated in both sensory modalities bilaterally. This region encompasses the VTA and extends laterally into the substantia nigra. A region in the left dorsolateral region of the superior medulla was activated solely during visceral pain. This region contains the nucleus tractus solitarius NTS), gracile nucleus (GN), and dorsoreticular nucleus, key regions in visceral and nociceptive processing. In several regions, areas of activation overlap. Despite this, separate peaks of activation are present in each area (Fig. 4, red nuclei and nucleus cuneiformis). In these cases, the voxel with the peak $z$-score has been used to identify the location of the individual nuclei. In addition, some of the nuclei discussed lie adjacent to each other (such as the PAG and NCF). Thus, even with higher resolution, clear demarcations between all nuclei would not be possible.

\section{ROI}

To assess whether the quantitative activation within brainstem regions differed between the visceral and somatic modalities, ROI analysis was performed. There were no differences in left or right PAG, VTA, dorsolateral pons (PBN-NC), or RVM activity between the two sensory modalities. However, both left and right NCF activity were significantly greater for visceral pain compared with somatic pain (left NCF, $p=0.02$; right $\mathrm{NCF}, p=0.01$; Student's paired $t$ test, two-tailed) (Fig. 5). Correlation analysis was performed between an individual's anxiety ratings and mean left and right PAG parameter estimate. No correlation was observed between somatic pain-induced left or right PAG activity and anxiety rating (left, $r=0.2, p=0.64$; right, $r=0.1, p=0.8$; Pearson's $r$, two-tailed). Right PAG activity significantly correlated with anxiety for visceral pain $(r=0.74 ; p<0.05$; Pearson's $r$, two-tailed) and approached significance for the left PAG $(r=$ $0.66 ; p=0.08$; Pearson's $r$, two-tailed) (Fig. 6). To assess whether increased anxiety in the visceral group contributed toward the greater NCF activation, subject anxiety ratings were correlated with NCF activity during visceral pain. No correlation was ob-

Table 1. Individual subject's activation during somatic and visceral pain

\begin{tabular}{|c|c|c|c|c|c|c|c|c|c|c|c|c|c|c|c|c|c|c|c|c|}
\hline Subject & 1 & & 2 & & 3 & & 4 & & 5 & & 6 & & 7 & & 8 & & 9 & & 10 & \\
\hline \multicolumn{21}{|l|}{ Somatic } \\
\hline Periaqueductal grey & B & 3.32 & $\mathrm{~L}$ & 2.45 & & 0.00 & $\mathrm{R}$ & 2.47 & & 0.40 & & 2.27 & & 1.43 & & 0.00 & B & 3.42 & & 1.61 \\
\hline Nucleus cuneiformis & $\mathrm{R}$ & 2.96 & B & 2.61 & & 0.00 & $\mathrm{R}$ & 2.94 & $\mathrm{R}$ & 2.33 & & 2.1 & & 0.84 & & 0.00 & $\mathrm{R}$ & 2.58 & $\mathrm{R}$ & 2.74 \\
\hline Red nucleus & $\mathrm{R}$ & 2.56 & B & 4.09 & & 0.00 & B & 2.74 & $\mathrm{R}$ & 2.68 & $\mathrm{R}$ & 2.91 & & 1.87 & & 0.21 & $\mathrm{R}$ & 2.97 & $\mathrm{R}$ & 3.19 \\
\hline Ventral tegmental area & B & 3.06 & $\mathrm{~L}$ & 2.89 & & 1.52 & & 1.56 & & 1.12 & B & 3.64 & & 1.87 & & 0.59 & L & 2.72 & $\mathrm{R}$ & 3.85 \\
\hline Dorsolateral pons & & 2.08 & B & 2.74 & & 0.00 & B & 2.84 & & 0.00 & & 1.49 & $\mathrm{R}$ & 2.65 & & 0.00 & B & 6.98 & B & 3.46 \\
\hline RVM & & 1.82 & M & 2.77 & & 0.00 & & 0.48 & & 0.00 & $\mathrm{R}$ & 3.08 & & 0.23 & & 0.00 & & 0.00 & & 0.74 \\
\hline Dorsolateral medulla & & 0.69 & & 1.62 & & 0.00 & & 1.48 & & 0.00 & $\mathrm{R}$ & 2.81 & & 1.58 & & 0.00 & & 0.07 & $\mathrm{R}$ & 2.98 \\
\hline \multicolumn{21}{|l|}{ Visceral } \\
\hline Periaqueductal grey & $\mathrm{L}$ & 2.98 & B & 4.93 & B & 3.09 & B & 2.33 & B & 5.07 & & 1.18 & & 1.39 & B & 2.93 & B & 6.67 & $\mathrm{R}$ & 2.36 \\
\hline Nucleus cuneiformis & $\mathrm{L}$ & 3.18 & B & 6.21 & B & 3.31 & B & 4.17 & B & 6.14 & & 1.80 & & 1.33 & B & 5.78 & & 1.89 & $\mathrm{R}$ & 2.65 \\
\hline Red nucleus & & 1.72 & B & 4.50 & B & 3.14 & B & 4.99 & B & 4.22 & B & 3.13 & & 1.52 & B & 6.35 & & 1.77 & $\mathrm{R}$ & 3.30 \\
\hline Ventral tegmental area & & 1.95 & B & 3.53 & B & 4.00 & $\mathrm{~L}$ & 3.82 & B & 4.79 & & 0.98 & & 1.72 & B & 8.09 & & 0.02 & B & 4.75 \\
\hline Dorsolateral pons & $\mathrm{L}$ & 3.08 & B & 7.22 & & 1.87 & B & 3.38 & B & 3.30 & & 0.00 & & 0.61 & B & 3.09 & B & 5.22 & B & 3.31 \\
\hline RVM & & 0.00 & M & 4.26 & & 0.00 & & 1.50 & B & 4.30 & & 0.00 & & 0.00 & & & M & 2.36 & & 1.54 \\
\hline Dorsolateral medulla & & 0.97 & & 1.75 & & 0.02 & B & 2.88 & $\mathrm{R}$ & 2.96 & & 0.00 & & 0.71 & B & 3.72 & B & 4.16 & $\mathrm{R}$ & 2.37 \\
\hline
\end{tabular}

For each subject (1-10), the peak $z$-score for each brainstem region is listed. When the $z$-score crossed the statistical threshold ( $z>2.3 ; p<0.01)$, the side is indicated ( $R$, right; $L$, left; $B$, bilateral; $M$, midline). 


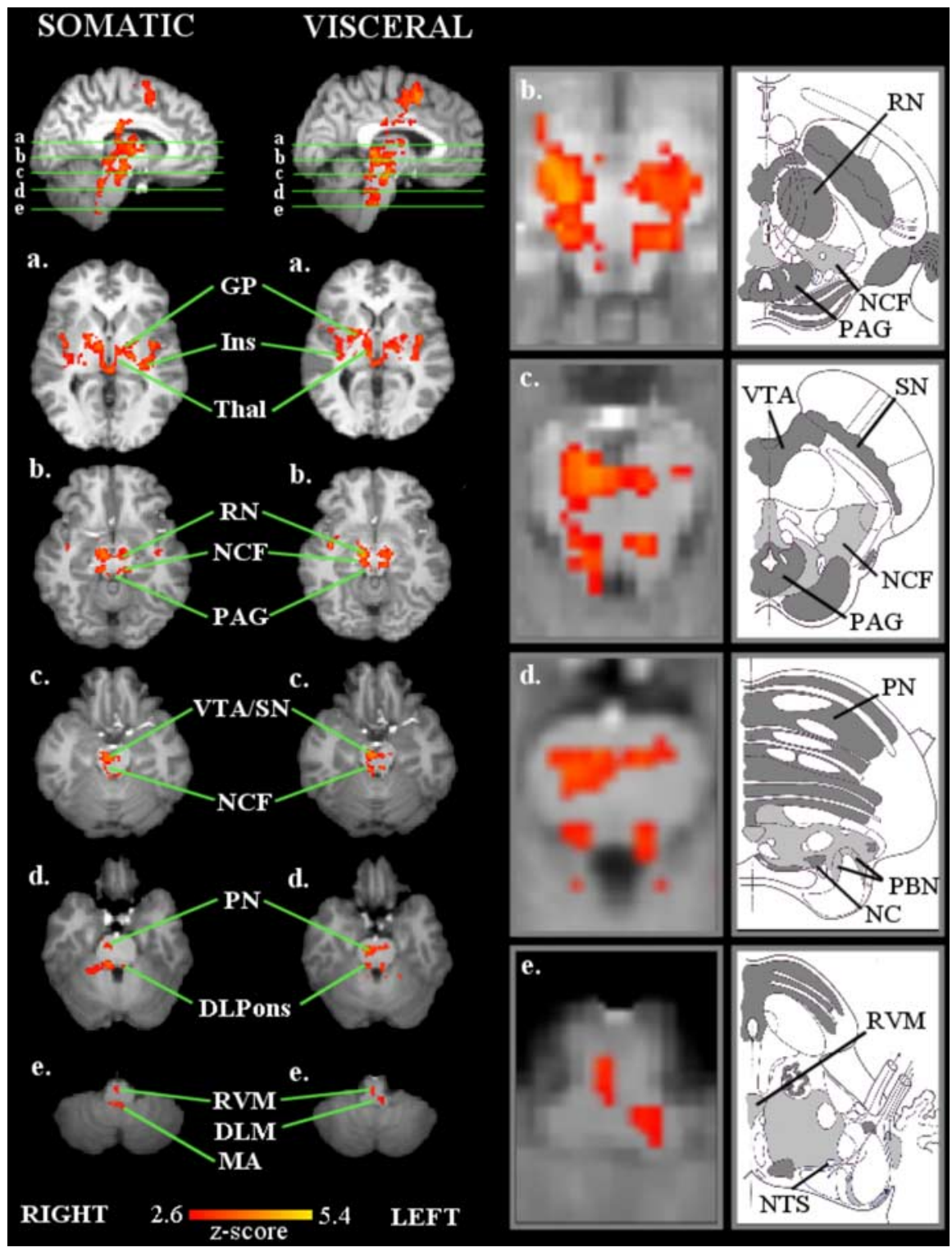

Figure 4. Group activation maps for somatic (first column) and visceral (second column) pain. The sagittal slices show columns of activation within the brainstem in each group. Axial slices (a-e) correspond to the plane indicated in the sagittal slice. A reduced field of view was used during functional scanning; thus, the activation map is limited in its anteroposterior plane. The two groups have a similar spatial pattern of activation. Regions commonly activated in whole-brain pain-imaging studies are significantly activated: thalamus (Thal) and posterior insula (Ins) bilaterally. Activation was also seen in the globus pallidus (GP) bilaterally in both groups. Significantly, activated brainstem nuclei included the red nucleus (RN), NCF, PAG, VTA [which extended laterally into the substantia nigra (SN)], and the dorsolateral pons (DLPons) bilaterally. Bilateral activation of the PN occurred in the visceral group but was limited to the right side in the somatic group. Activation in the region of the RVM was also seen in both groups. In the visceral group alone, a region of activation occurred in the left dorsolateral medulla (DLM). A small area of motion artifact (MA) occurred around the central canal in the somatic group. The axial slices in the visceral group have been magnified and juxtaposed to drawings at the same anatomical location [modified from Duvernoy (1995) with permission]. strated a consistent correlation between RVM activity and that of the dorsolateral pons only. A significant correlation was seen bilaterally for visceral stimulation (right, $r=0.65, p=0.04$; left, $r=0.75, p=$ 0.01; Pearson's $r$, two-tailed) but only on the left side for somatic stimulation (right, $r=0.51, p=0.13$; left, $r=0.75, p=0.03$; Pearson's $r$, two-tailed) (Fig. 7).

Because the stimuli used were midline, we would expect any significant effects in the ROI analysis to be bilateral. This is the case for NCF parameter estimate comparison between visceral and somatic pain and correlations of dorsolateral pons and RVM activity during visceral pain. Likewise, the correlation of PAG activity with anxiety during visceral pain shows a trend toward a bilaterally significant result. We contend that this dramatically reduces the chances that these effects are being falsely reported as positive. These values have therefore not been corrected for multiple comparisons because use of Bonferroni's correction would be excessively stringent.

\section{Discussion}

The results from this study demonstrate that it is feasible to perform an fMRI of the brainstem (from medulla to mesencephalon) in individual subjects, thus supporting previous studies (Komisaruk et al., 2002; Tracey et al., 2002; Topolovec et al., 2004). Detailed attention to accurate brain segmentation and registration has allowed us to further these observations by performing group analyses. These have shown bilateral regional brainstem activation in areas consistent with the PAG, RVM, NCF, and the dorsolateral pons. Furthermore, the pattern of spatial activation is similar for the two sensory modalities, visceral and somatic. These results are consistent with animal stimulation and tracing studies (Traub et al., 1996; Rodella et al., 1998; Millan, 2002; Monnikes et al., 2003) and the marked viscerosomatic convergence that occurs at the spinal cord level (Ruch, 1946). The main spatial difference between the two sensory modalities is activation of the dorsolateral medulla (including the gracile nucleus) in the visceral group only. We also identified differences in behavior of individual regions: greater quanserved (right NCF, $r=-0.02, p>0.05$; left NCF, $r=0.19, p>$ 0.05; Pearson's $r$, two-tailed). To establish whether a behavioral response (i.e., anxiety) altered either visceral or somatic pain thresholds (as the perception was locked, and top-down influence on the current needed to drive the same perception of pain might have occurred), anxiety rating was correlated with delivered current. No significant correlation was observed for either somatic or visceral pain (somatic, $r=0.41, p>0.05$; visceral, $r=$ $0.05, p>0.05$; Pearson's $r$, two-tailed).

Additional analysis of the regional brainstem activity demon- titative activation bilaterally in the NCF and a correlation of anxiety with right PAG activity during visceral pain alone.

\section{Nucleus cuneiformis}

Generally, we do not feel peristalsis (passage of stool or gas), yet in rodents visceral afferents are excited by non-noxious, physiological stimuli (Andrew and Blackshaw, 2001), and subliminal visceral stimulation in humans results in cortical activation, as seen with fMRI (Kern and Shaker, 2002). Therefore, information 


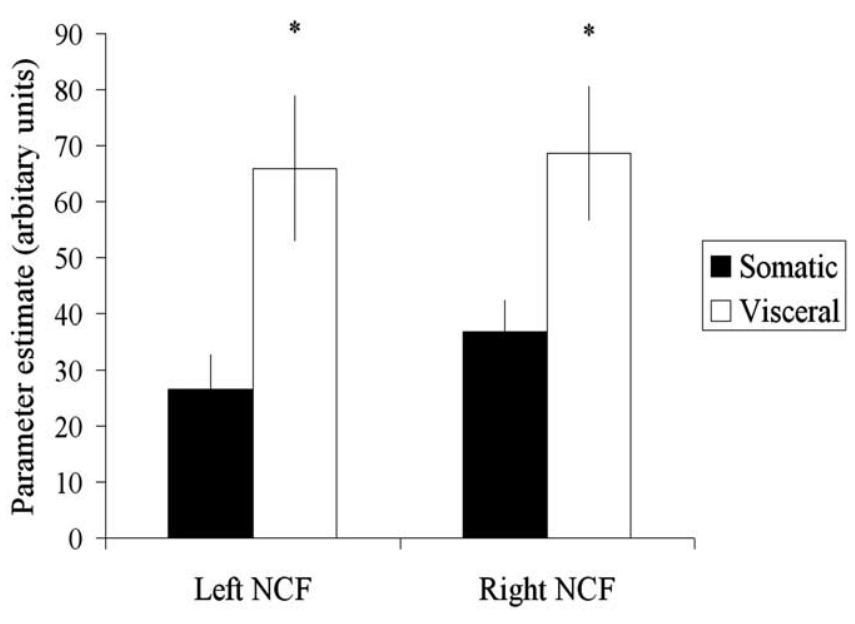

Figure 5. Nucleus cuneiformis activity. Significantly greater activation occurred bilaterally in the NCF during visceral pain ( ${ }^{*} p<0.05$; Student's $t$ test, two-tailed, paired, uncorrected). Error bars represent $\mathrm{SE}$.

must be reaching the dorsal horn or brainstem (via the vagus nerve). Why then do we not, in general, perceive the normal functioning of our gastrointestinal tracts? One possible explanation is that visceral input could be under a persistent inhibitory control. Undoubtedly, constant perception of gut activity would act as a considerable distraction to daily activity. Our results have shown that the NCF, which is known to have nocifensive functions, is activated to a significantly greater degree during visceral pain than somatic pain. Therefore, visceral pain may result in a heightened, descending inhibitory control from brainstem centers such as the NCF, thus preventing sufficient input to the cortex to allow conscious perception of afferent information from the bowel. The NCF, a nucleus of the brainstem reticular formation contains functionally distinct classes of neurons that are able to enhance nociception ("ON cells") and inhibit nociception ("OFF cells") (Haws et al., 1989), characteristics it shares with the PAG and RVM (Fields et al., 1983; Heinricher et al., 1987). It has strong neuronal connections with the PAG, RVM, PFC, hypothalamus, and amygdala (Edwards and de Olmos, 1976; Zemlan and Behbehani, 1988; Bernard et al., 1989; Sesack et al., 1989), and c-Fos immunoreactivity in this region has been induced by colorectal distension in rats (Monnikes et al., 2003). Therefore, it is tactically positioned to receive emotive, autonomic, and executive inputs, in concert with the PAG and RVM, and can therefore influence nociceptive processing in a facilitatory or inhibitory manner. Indeed, a recent human fMRI study of mechanical stimulation to experimentally induced secondary hyperalgesia demonstrated a significantly greater left NCF activation compared with mechanical stimulation in the control stimulation condition, thus illustrating its potential facilitatory role in the development of central sensitization (Zambreanu et al., 2005). The absence of a correlation with anxiety ratings suggests that the increased NCF activity seen in the visceral group is not related to the heightened anxiety observed.

\section{Periaqueductal gray}

Subjects experienced higher anxiety during the visceral stimulation compared $p=0.08$; Pearson's $r$, two-tailed). with somatic stimulation. Furthermore, anxiety ratings correlated with right PAG activation during visceral but not somatic pain (Fig. 6). Anxiety and the stress response are coordinated through the PAG together with other regions such as the hypothalamus and amygdala. William James in 1884 proposed that emotions are dictated by and reliant on bodily sensations (James, 1884). Modern proponents of these theories comment that the physical sensation of anxiety, or any emotional response, is dependent on sensations from the viscera (Damasio, 1996). This has presumably led to the aphorisms "gut feelings," "butterflies in the stomach," and "a visceral response." These emotively induced visceral sensations may be attributable to changes in autonomic outflow and resultant increased gut tone (Almy et al., 1949; Whorwell et al., 1992). However, the consequential visceral sensation induced by these changes in gut tone will transmit emotive meaning. Therefore, the correlation of visceral, but not somatic, pain-induced PAG activity with anxiety ratings could represent the neural correlates of visceral-based perception of anxiety [i.e. peripheral visceral signals preferentially (over somatic inputs) connect with the PAG to contribute to the sensation of anxiety perceived by the subject].

These theories are speculative, alternative explanations are possible. For example, pain perception can be increased through high anxiety (Ploghaus et al., 2001) or decreased through stressinduced analgesia (Fields, 2000). Thus, the correlation of right PAG activity with visceral pain may reflect an emotively/autonomically induced descending modulation of the noiceptive input. It is impossible to ascertain whether this modulation is facilitatory or inhibitory in our results as the pain intensity of the stimuli was locked.

\section{Dorsolateral medulla}

An additional difference in the observed activations was a region of activation in the left dorsolateral medulla in the visceral group only (Fig. 4). This area includes important, nociceptive nuclei: the NTS and the GN. The NTS receives a predominantly visceral input (nociceptive and non-nociceptive) from the vagal nerve and spinal afferents. Its most caudal aspect, extending the length of the medulla, receives input from the visceral organs and vasculature, with the superior pole receiving gustatory neurons. Electrical stimulation of the nucleus can induce both inhibition (Aicher and Randich, 1990) and facilitation (via the vagal nerve) (Randich and Gebhart, 1992) of nociception in rodents. It therefore would be expected to contribute to the measured activation in response to nociceptive visceral stimulation, as found in our study. The GN receives a mainly visceral nociceptive input from the dorsal column pathway (Willis et al., 1999). It is thought to represent an ascending part of an amplification loop that, when activated, heightens visceral nociceptive perception possibly, al-
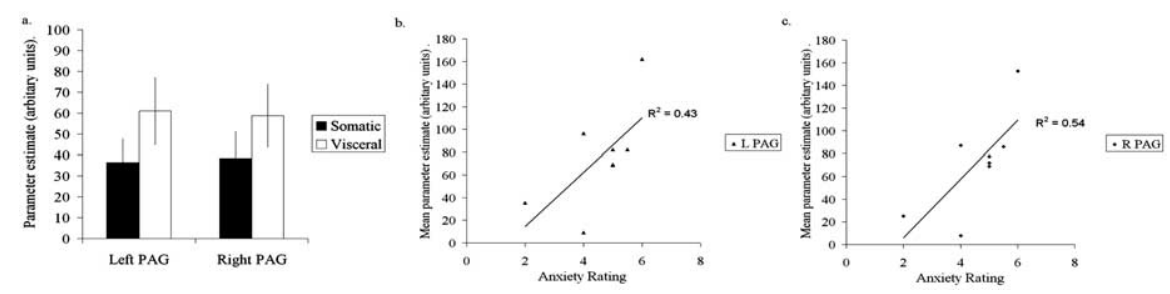

Figure 6. Correlation of anxiety with PAG activity during visceral pain. $\boldsymbol{a}$, The mean PAG parameter estimate for visceral and somatic pain; no significant difference in activation was observed. $\boldsymbol{a}, \boldsymbol{b}$, Anxiety during the painful visceral stimuli correlated with right (R) PAG activity $(\boldsymbol{b} ; r=0.74 ; p<0.05$; Pearson's $r$, two-tailed) and approached significance for the left (L) PAG $(\boldsymbol{a} ; r=0.66$; 
a.
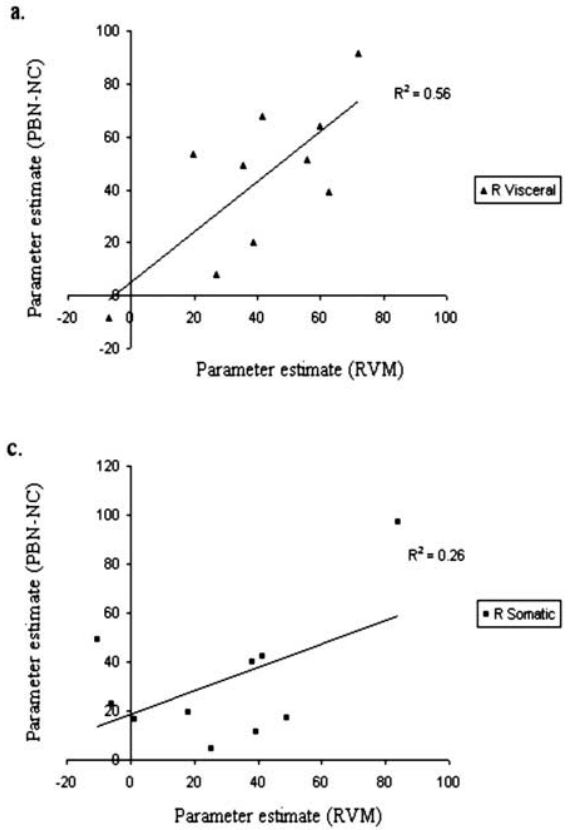
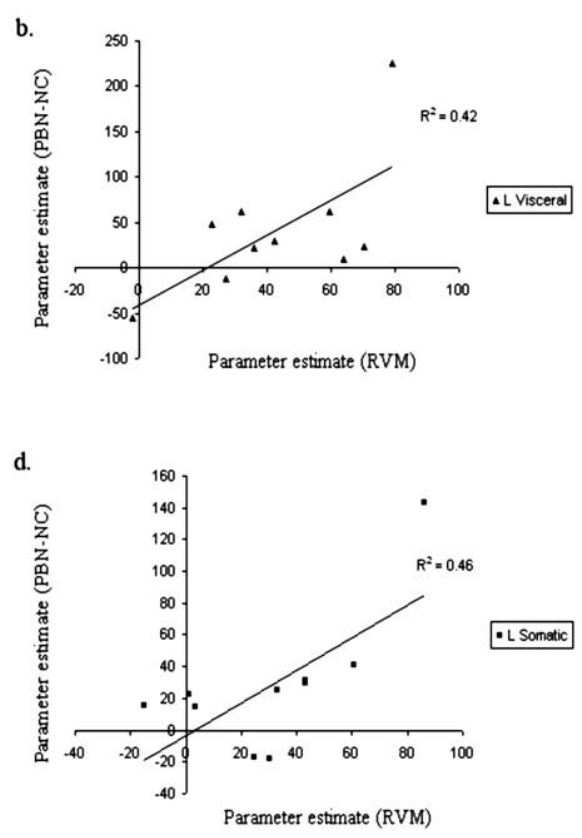

Figure 7. Correlation analysis between RVM activity and that of the dorsolateral pons in the region of the PBN and NC (PBNNC). A significant correlation was seen bilaterally for visceral stimulation $(\boldsymbol{a}, \boldsymbol{b})$ (right, $r=0.65, p=0.04$; left, $r=0.75, p=0.01$; Pearson's $r$, two-tailed) but not for somatic stimulation $(\boldsymbol{c}, \boldsymbol{d})$ (right, $r=0.51, p=0.13$; left, $r=0.75, p=0.03$; Pearson's $r$, two-tailed). R, Right; L, left

though facilitatory influences of the RVM (Palecek, 2004). Another nucleus found in this region of activation is the dorsal reticular nucleus of the medulla. This receives both visceral and somatic input and is both pronociceptive and nocifensive (Lima and Almeida, 2002). Studies suggest that this area is crucial for the development of diffuse noxious inhibitory controls (Villanueva and Le Bars, 1995). Future high spatial-resolution studies will aim to resolve these structures functionally to determine their separate contributions to visceral nociceptive processing.

\section{Ventral tegmental area}

These results also suggest a role for the VTA in both visceral and somatic pain processing. This region has been studied extensively in studies of addiction and reward in human and animal studies and represents part of the mesolimbic reward circuitry. It has close connections with the limbic system, especially the cingulate cortex (Oades and Halliday, 1987), and rodent studies have shown that connectivity exists with the PAG and RVM (Kirouac et al., 2004). Electrical stimulation induces an antinociceptive effect in rats (Sotres-Bayon et al., 2001). Furthermore, nondopaminergic neurons exist within the VTA that respond to aversive, not rewarding stimuli (Ungless et al., 2004), suggesting a role of the VTA in the affective/aversive dimension of pain processing.

\section{RVM: dorsolateral pons correlation of activation}

A significant correlation between left and right dorsolateral pons (incorporating the PBN and NC) and RVM activity occurred during visceral pain. The spinoparabrachial pathway provides a major tract for ascending nociceptive information (Hunt and Mantyh, 2001). More than $80 \%$ of lamina 1 dorsal horn projection neurons to the PBN express the neurokinin-1 (NK-1) receptor (Todd et al., 2000). Selective ablation of lamina 1 neurons with NK-1 receptors results in a marked attenuation of capsaicininduced thermal and mechanical primary and secondary hyper- algesia in rodents (Mantyh et al., 1997; Suzuki et al., 2002). Inactivation of the RVM can abolish the development of secondary hyperalgesia in rodents (Urban and Gebhart, 1999). A spino-bulbo-spinal loop to and from lamina 1 to the PBN and back to the RVM to either facilitate or inhibit nociceptive input at the level of the dorsal horn has been postulated (Suzuki et al., 2004). This loop could explain the correlation observed in our study. Alternatively, this observed correlation may be attributable to similar activity of the NC and RVM. The NC also has descending inhibitory functions, electrical stimulation induces antinociception in the rat (Jones and Gebhart, 1986). Therefore, descending nociceptive inhibition may be occurring in parallel with the RVM.

At both an individual (Table 1) and group (Fig. 4) level, we observed areas of activation within the brainstem, the locality of which correlate with regions known to be involved in pain processing in animals (Traub et al., 1996; Rodella et al., 1998; Millan, 2002; Monnikes et al., 2003) and human studies (Boivie and Meyerson, 1982; Baskin et al., 1986; Tracey et al., 2002). The spatial pattern of activation was very similar for visceral and somatic pain; however, greater quantitative bilateral NCF activity during visceral pain compared with somatic was found. Furthermore, correlation of right PAG activity with anxiety was observed only during visceral pain. We propose that these findings may underlie some of the perceptual and behavioral differences observed between the two sensory modalities. Noninvasive tools are essential if we are to gain additional understanding of the complex interactions of the human brainstem nuclei (Iannetti and Tracey, 2005), not only in pain, but for all sensory, autonomic, and motor functions in humans in health and disease.

\section{References}

Aicher SA, Randich A (1990) Antinociception and cardiovascular responses produced by electrical stimulation in the nucleus tractus solitarius, nucleus reticularis ventralis, and the caudal medulla. Pain 42:103-119.

Almy T, Kern F, Tulin M (1949) Alteration in colonic function in man under stress. II. Experimental production of sigmoid spasm in healthy persons. Gastroenterology 12:425-436.

Andrew LK, Blackshaw LA (2001) Colonic mechanoreceptor inputs to rat lumbo-sacral dorsal horn neurones: distribution, thresholds and chemosensory modulation. Neurogastroenterol Motil 13:333-337.

Bantick SJ, Wise RG, Ploghaus A, Clare S, Smith SM, Tracey I (2002) Imaging how attention modulates pain in humans using functional MRI. Brain 125:310-319.

Basbaum AI, Clanton CH, Fields HL (1976) Opiate and stimulus-produced analgesia: functional anatomy of a medullospinal pathway. Proc Natl Acad Sci USA 73:4685-4688.

Baskin DS, Mehler WR, Hosobuchi Y, Richardson DE, Adams JE, Flitter MA (1986) Autopsy analysis of the safety, efficacy and cartography of electrical stimulation of the central gray in humans. Brain Res 371:231-236

Beck AT, Ward CH, Mendelson M, Mock J, Erbaugh J (1961) An inventory for measuring depression. Arch Gen Psychiatry 4:561-571.

Beecher HK (1946) Pain in men wounded in battle. Ann Surg 123:96-105. 
Bernard JF, Peschanski M, Besson JM (1989) Afferents and efferents of the rat cuneiformis nucleus: an anatomical study with reference to pain transmission. Brain Res 490:181-185.

Boivie J, Meyerson BA (1982) A correlative anatomical and clinical study of pain suppression by deep brain stimulation. Pain 13:113-126.

Brodal P (1968) The corticopontine projection in the cat. I. Demonstration of a somatotopically organized projection from the primary sensorimotor cortex. Exp Brain Res 5:210-234.

Damasio AR (1996) Descartes error: emotion, reason and the human brain. London: Papermac.

Duvernoy H (1995) The human brainstem and cerebellum. New York: Springer/Wien.

Edwards SB, de Olmos JS (1976) Autoradiographic studies of the projections of the midbrain reticular formation: ascending projections of nucleus cuneiformis. J Comp Neurol 165:417-431.

Fields HL (2000) Pain modulation: expectation, opioid analgesia and virtual pain. Prog Brain Res 122:245-253.

Fields HL, Basbaum AI, Clanton CH, Anderson SD (1977) Nucleus raphe magnus inhibition of spinal cord dorsal horn neurons. Brain Res 126:441-453.

Fields HL, Bry J, Hentall I, Zorman G (1983) The activity of neurons in the rostral medulla of the rat during withdrawal from noxious heat. J Neurosci 3:2545-2552.

Friston KJ, Worsley KJ, Frackowiak RS, Maziotta JC, Evans AC (1994) Assessing the significance of focal activations using their spatial extent. Hum Brain Mapp 1:214-220.

Gebhart GF (2004) Descending modulation of pain. Neurosci Biobehav Rev 27:729-737.

Haws CM, Williamson AM, Fields HL (1989) Putative nociceptive modulatory neurons in the dorsolateral pontomesencephalic reticular formation. Brain Res 483:272-282.

Heinricher MM, Cheng ZF, Fields HL (1987) Evidence for two classes of nociceptive modulating neurons in the periaqueductal gray. J Neurosci 7:271-278.

Hunt SP, Mantyh PW (2001) The molecular dynamics of pain control. Nat Rev Neurosci 2:83-91.

James W (1884) What is an emotion? Mind 9:188-205.

Jenkinson M, Bannister P, Brady M, Smith S (2002) Improved optimisation for the robust and accurate linear registration and motion correction of brain images. NeuroImage 17:825-841.

Jones SL, Gebhart GF (1986) Quantitative characterization of ceruleospinal inhibition of nociceptive transmission in the rat. J Neurophysiol 56:1397-1410.

Kern MK, Shaker R (2002) Cerebral cortical registration of subliminal visceral stimulation. Gastroenterology 122:290-298.

Kirouac GJ, Li S, Mabrouk G (2004) GABAergic projection from the ventral tegmental area and substantia nigra to the periaqueductal gray region and the dorsal raphe nucleus. J Comp Neurol 469:170-184.

Komisaruk BR, Mosier KM, Liu WC, Criminale C, Zaborszky L, Whipple B, Kalnin A (2002) Functional localization of brainstem and cervical spinal cord nuclei in humans with fMRI. Am J Neuroradiol 23:609-617.

Leergaard TB, Alloway KD, Pham TA, Bolstad I, Hoffer ZS, Pettersen C, Bjaalie JG (2004) Three-dimensional topography of corticopontine projections from rat sensorimotor cortex: comparisons with corticostriatal projections reveal diverse integrative organization. J Comp Neurol 478:306-322.

Lima D, Almeida A (2002) The medullary dorsal reticular nucleus as a pronociceptive centre of the pain control system. Prog Neurobiol 66:81-108.

Mantyh PW, Rogers SD, Honore P, Allen BJ, Ghilardi JR, Li J, Daughters RS, Lappi DA, Wiley RG, Simone DA (1997) Inhibition of hyperalgesia by ablation of lamina I spinal neurons expressing the substance P receptor. Science 278:275-279.

Millan MJ (2002) Descending control of pain. Prog Neurobiol 66:355-474.

Miron D, Duncan GH, Bushnell MC (1989) Effects of attention on the intensity and unpleasantness of thermal pain. Pain 39:345-352.

Monnikes H, Ruter J, Konig M, Grote C, Kobelt P, Klapp BF, Arnold R, Wiedenmann B, Tebbe JJ (2003) Differential induction of c-fos expression in brain nuclei by noxious and non-noxious colonic distension: role of afferent $\mathrm{C}$-fibers and 5-HT3 receptors. Brain Res 966:253-264.
Oades RD, Halliday GM (1987) Ventral tegmental (A10) system: neurobiology. 1. Anatomy and connectivity. Brain Res 434:117-165.

Ogawa S, Tank DW, Menon R, Ellermann JM, Kim SG, Merkle H, Ugurbil K (1992) Intrinsic signal changes accompanying sensory stimulation: functional brain mapping with magnetic resonance imaging. Proc Natl Acad Sci USA 89:5951-5955.

Palecek J (2004) The role of dorsal columns pathway in visceral pain. Physiol Res 53 [Suppl 1]:S125-S130.

Ploghaus A, Narain C, Beckmann CF, Clare S, Bantick S, Wise R, Matthews PM, Rawlins JN, Tracey I (2001) Exacerbation of pain by anxiety is associated with activity in a hippocampal network. J Neurosci 21:9896-9903.

Porreca F, Ossipov MH, Gebhart GF (2002) Chronic pain and medullary descending facilitation. Trends Neurosci 25:319-325.

Randich A, Gebhart GF (1992) Vagal afferent modulation of nociception. Brain Res Rev 17:77-99.

Reynolds DV (1969) Surgery in the rat during electrical analgesia induced by focal brain stimulation. Science 164:444-445.

Rodella L, Rezzani R, Gioia M, Tredici G, Bianchi R (1998) Expression of Fos immunoreactivity in the rat supraspinal regions following noxious visceral stimulation. Brain Res Bull 47:357-366.

Ruch TC (1946) Visceral sensation and referred pain. In: Howell's textbook of physiology, Ed 15 (Fulton JF, ed), pp 385-401. Philadelphia: Saunders.

Sesack SR, Deutch AY, Roth RH, Bunney BS (1989) Topographical organization of the efferent projections of the medial prefrontal cortex in the rat: an anterograde tract-tracing study with Phaseolus vulgaris leucoagglutinin. J Comp Neurol 290:213-242.

Sherrington C 1906 The integrative action of the nervous system. Oxford: Oxford UP.

Smith SM (2002) Fast robust automated brain extraction. Hum Brain Mapp 17:143-155.

Smith SM, De-Stefano N, Jenkinson M, Matthews PM (2001) Normalized accurate measurement of longitudinal brain change. J Comput Assist Tomogr 25:466-475

Sotres-Bayon F, Torres-Lopez E, Lopez-Avila A, del Angel R, Pellicer F (2001) Lesion and electrical stimulation of the ventral tegmental area modify persistent nociceptive behavior in the rat. Brain Res 898:342-349.

Suzuki R, Morcuende S, Webber M, Hunt SP, Dickenson AH (2002) Superficial NK1-expressing neurons control spinal excitability through activation of descending pathways. Nat Neurosci 5:1319-1326.

Suzuki R, Rygh LJ, Dickenson AH (2004) Bad news from the brain: descending 5-HT pathways that control spinal pain processing. Trends $P$ harmacol Sci 25:613-617.

Talairach J, Tournoux P (1988) Co-planar stereotaxic atlas of the human brain, pp 1-222. New York: Thieme Medical Publishers.

Talley NJ, Phillips SF, Melton III J, Wiltgen C, Zinsmeister AR (1989) A patient questionnaire to identify bowel disease. Ann Intern Med 111:671-674.

Todd AJ, McGill MM, Shehab SA (2000) Neurokinin 1 receptor expression by neurons in laminae I, III and IV of the rat spinal dorsal horn that project to the brainstem. Eur J Neurosci 12:689-700.

Topolovec JC, Gati JS, Menon RS, Shoemaker JK, Cechetto DF (2004) Human cardiovascular and gustatory brainstem sites observed by functional magnetic resonance imaging. J Comp Neurol 471:446-461.

Tracey I, Iannetti G (2005) Functional imaging of the human brainstem. Clin Neurophysiol, in press.

Tracey I, Ploghaus A, Gati JS, Clare S, Smith S, Menon RS, Matthews PM (2002) Imaging attentional modulation of pain in the periaqueductal gray in humans. J Neurosci 22:2748-2752.

Traub RJ, Silva E, Gebhart GF, Solodkin A (1996) Noxious colorectal distention induced-c-Fos protein in limbic brain structures in the rat. Neurosci Lett 215:165-168.

Ungless MA, Magill PJ, Bolam JP (2004) Uniform inhibition of dopamine neurons in the ventral tegmental area by aversive stimuli. Science 303:2040-2042.

Urban MO, Gebhart GF (1997) Characterization of biphasic modulation of spinal nociceptive transmission by neurotensin in the rat rostral ventromedial medulla. J Neurophysiol 78:1550-1562.

Urban MO, Gebhart GF (1999) Supraspinal contributions to hyperalgesia. Proc Natl Acad Sci USA 96:7687-7692. 
Villanueva L, Le Bars D (1995) The activation of bulbo-spinal controls by peripheral nociceptive inputs: diffuse noxious inhibitory controls. Biol Res 28:113-125.

Whorwell PJ, Houghton LA, Taylor EE, Maxton DG (1992) Physiological effects of emotion: assessment via hypnosis. Lancet 340:69-72.

Willis WD, Al-Chaer ED, Quast MJ, Westlund KN (1999) A visceral pain pathway in the dorsal column of the spinal cord. Proc Natl Acad Sci USA 96:7675-7679.

Woolrich MW, Ripley BD, Brady J, Smith SM (2001) Temporal autocorrelation in univariate linear modelling of FMRI data. NeuroImage 14:1370-1386.
Worsley KJ, Evans AC, Marrett S, Neelin P (1992) A three-dimensional statistical analysis for $\mathrm{CBF}$ activation studies in human brain. J Cereb Blood Flow Metab 12:900-918.

Zambreanu L, Wise RG, Brooks JCW, Iannetti GD, Tracey I (2005) A role for the brainstem in central sensitisation in humans. Evidence from functional magnetic resonance imaging. Pain 114:397-407.

Zemlan FP, Behbehani MM (1988) Nucleus cuneiformis and pain modulation: anatomy and behavioral pharmacology. Brain Res 453:89-102.

Zhuo M, Gebhart GF (1990) Characterization of descending inhibition and facilitation from the nuclei reticularis gigantocellularis and gigantocellularis pars alpha in the rat. Pain 42:337-350. 